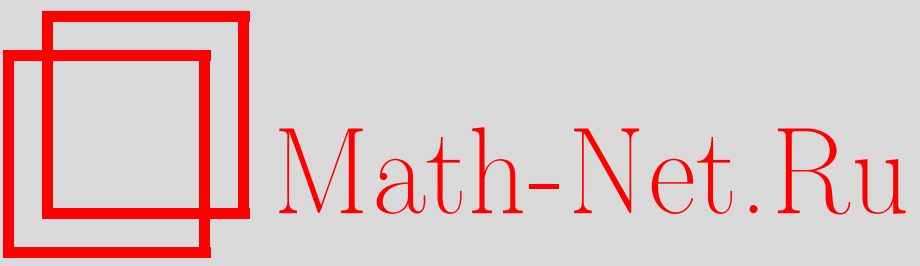

М. М. Гриненко, О послойных перестройках расслоений на поверхности дель Пеццо степени 2, УМН, 2001, том 56, выпуск 4, 145-146

DOI: https://doi.org/10.4213/rm425

Использование Общероссийского математического портала Math-Net.Ru подразумевает, что вы прочитали и согласны с пользовательским соглашением

http://www . mathnet.ru/rus/agreement

Параметры загрузки:

IP : 18.234 .197 .8

26 апреля 2023 г., 17:43:43 


\title{
О ПОСЛОЙНЫХ ПЕРЕСТРОЙКАХ РАССЛОЕНИЙ НА ПОВЕРХНОСТИ ДЕЛЬ ПЕЦЦО СТЕПЕНИ 2
}

\author{
М. М. ГРИНЕНКО
}

Всюду предполагается, что основное поле алгебраически замкнуто и имеет характеристику 0 . Встречающиеся определения и понятия (бирациональная жесткость, Мори-расслоение и т. п.) читатель может найти в [1] и [2].

Пусть $S$ - гладкая алгебраическая кривая (не обязательно полная) с отмеченной точкой $O$, $X$ и $V$ - гладкие трехмерные многообразия, обладающие сюръективными проективными морфизмами $\pi: X \rightarrow S$ и $\pi^{\prime}: V \rightarrow S$, все слои которых - поверхности дель Пецщо степени 2. Центральные слои (т.е. слои над точкой $O$ ) обозначим $X_{0}$ и $V_{0}$ соответственно. Предположим, что задана коммутативная диаграмма

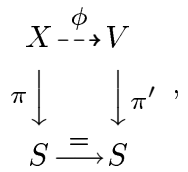

где $\phi$ - бирациональное отображение, являющееся изоморфизмом вне централных слоев.

ПРЕДЛОЖЕНИЕ. $\phi$ продолжается до изоморфизма $X u V$.

Важньм следствием этого предложения является утверждение о единственности гладкой модели, усиливающее результаты работы [1]:

СледствиЕ. Пусть $V \rightarrow \mathbb{P}^{1}-$ Мори-расслоение на поверхности дель Пеццо степени 2. Тогда класс Мори-расслоений, бирациональных над базой указанному $V / \mathbb{P}^{1}$, содержит не более одного гладкого әлемента. В частности, если $V / \mathbb{P}^{1}$ бирационально жестко и неособо, то это единственное неособое Мори-расслоение в своем классе бирациональной әквивалентности.

Аналогичное утверждение для степени 1 доказано в [2].

ДоКАЗАТЕЛЬСТВо ПРЕДЛОЖЕНИЯ. Предположим противное, т.е. что $\phi$ не изоморфизм. Мы можем полагать, что $S=\operatorname{Spec~} \mathscr{O}$, где $\mathscr{O}$ - локальное кольц с параметром $t$ (т.е. $t \mathscr{O}$ есть максимальный идеал, уравнение $t=0$ задает точку $O$ на $S$ ). Через $K$ обозначим поле частных кольца $\mathscr{O}$, через $\eta$ - общую схемную точку $S$. В дальнейшем индекс $\eta$ в обозначении многообразий означает локализацию в общей схемной точке $S$.

По условиям предложения, все слои - горенштейновы поверхности дель Пещо степени 2, а значит, обладают теми же свойствами проективного вложения, что и неособая поверхность дель Пеццо степени 2 [3], [4]. Таким образом, все они вкладьваются во взвешенное проективное пространство $\mathbb{P}(1,1,1,2)$ как поверхности степени 4 . Обозначая через $P_{1}$ и $P_{2}$ два экземпляра пространства $\mathbb{P}_{S}(1,1,1,2)$ над $S$, мы видим, что $X$ и $V$ могут рассматриваться как гиперповерхности в $P_{1}$ и $P_{2}$ соответственно, причем не пересекающие множества особых точек этих пространств ввиду условия неособости.

Очевидно, $\phi$ индуцирует изоморфизм $X_{\eta}$ и $V_{\eta}$, а значит, и изоморфизм между $P_{1_{\eta}}$ и $P_{2_{\eta}}$. Из элементарных линейно-алгебраических рассуждений следует, что мы можем выбрать координаты $[x, y, z, w]$ и $[p, q, r, s]$ с весами $(1,1,1,2)$ в $P_{1_{\eta}}$ и $P_{2_{\eta}}$ соответственно так, что $\phi$ и $\phi^{-1}$ могут быть записаны в виде

$$
\phi=\left\{\begin{array}{l}
p=t^{a} x \\
q=t^{b} y \\
r=t^{c} z \\
s=t^{d} w
\end{array}\right\}, \quad \phi^{-1}=\left\{\begin{array}{c}
x=t^{\alpha} p \\
y=t^{\beta} q \\
z=t^{\gamma} r \\
w=t^{\delta} s
\end{array}\right\},
$$

Работа вьполнена при поддержке Российского фонда фундаментальных исследований (гранты №№ 99-01-01132, 00-15-96085), грантов NWO-RFBR 047-008-005 и INTAS 97/2072. 
причем каждый из наборов целых чисел $(a, b, c, d)$ и $(\alpha, \beta, \gamma, \delta)$ содержит хотя бы один 0 и, более того, существует такое целое $m>0$, что

$$
m=a+\alpha=b+\beta=c+\gamma=\frac{1}{2}(d+\delta) .
$$

Для определенности мш будем полагать, что $\gamma=0$ (а значит, $c=m$ ) и $d \leqslant m / 2$.

Кроме того, координаты могут быть выбраны так, что $X$ и $V$ задаются уравнениями $w^{2}+$ $f_{4}(x, y, z)=0$ и $s^{2}+g_{4}(p, q, r)=0$ соответственно, где $f_{4}$ и $g_{4}$ - однородные полиномы степени 4 с коэффициентами в $\mathscr{O}$. Заметим, что коэффициенты при $w^{2}$ и $s^{2}$ могут быть выбраны равными 1 ввиду неособости многообразий.

Если мы теперь выразим координаты в уравнении для $V$ при помощи $\phi$, то после деления на коэффициент при $w^{2}$ (т.е. $t^{2 a}$ ) получим уравнение для $X$

$$
w^{2}+\sum_{\substack{i+j_{k}=4 \\ k \leqslant 2}} a_{i j k} x^{i} y^{j} z^{k}+t^{3 m-2 a} \sum_{i+j=1} b_{i j} x^{i} y^{j} z^{3}+c t^{4 m-2 a} z^{4}=0
$$

причем все коэффициенты (т.е. $a_{i j k}, b_{j i}$ и $c$ ) лежат в кольце $\mathscr{O}$. Элементарная проверка показывает, что точка (0: $0: 1: 0)$ должна быть особой. Полученное противоречие доказьвает предложение.

Для доказательства следствия достаточно заметить, что в заданной ситуации любое бирациональное над базой отображение "откручивается" при помощи бирациональных автоморфизмов $X$ (инволюции Бертини) до бирационального отображения, индуцирующего изоморфизм слоев над общими точками ([5; теорема 2.6$])$, и применить доказанное предложение.

ЗАмечАниЕ. Результат, сформулированньй в предложении, доказан в работе [6] при более сильных ограничениях (именно, когда центральный слой неособый) при степени слоя не вьше 4.

\section{СПИСОК ЛИТЕРАТУРЫ}

[1] М. М. Гриненко // Матем. сб. 2000. Т. 191. № 5. С. 17-38. [2] М. М. Гриненко // Матем. заметки. 2001. T. 69. № 4. C. 550-565. [3] F. Hidaka, K.-I. Watanabe // Tokyo J. Math. 1981. V. 4. № 2. P. 319-330. [4] M. Reid// Publ. Res. Inst. Math. Sci. 1994. V. 30. № 5. P. 695-727. [5] В. А. Исковских // УМН. 1996. Т. 51. № 4. С. 3-72. [6] J. Park. Birational maps of del Pezzo fibrations // math.AG/9912076. 